\title{
Decreased Brain pH as a Shared Endophenotype of Psychiatric Disorders
}

\begin{abstract}
Hideo Hagihara', Vibeke S Catts ${ }^{2,3}$, Yuta Katayama ${ }^{4}$, Hirotaka Shoji ', Tsuyoshi Takagi ${ }^{5,6}$, Freesia L Huang ${ }^{7}$, Akito Nakao', Yasuo Mori ${ }^{8}$, Kuo-Ping Huang ${ }^{7}$, Shunsuke Ishii ${ }^{6}$, Isabella A Graef', Keiichi I Nakayama ${ }^{4}$, Cynthia Shannon Weickert ${ }^{2,3}$ and Tsuyoshi Miyakawa*,I

'Division of Systems Medical Science, Institute for Comprehensive Medical Science, Fujita Health University, Toyoake, Japan; ${ }^{2}$ Schizophrenia Research Laboratory, Neuroscience Research Australia, Randwick, NSW, Australia; ${ }^{3}$ School of Psychiatry, University of New South Wales, Sydney, NSW, Australia; ${ }^{4}$ Department of Molecular and Cellular Biology, Medical Institute of Bioregulation, Kyushu University, Fukuoka, Japan; ${ }^{5}$ nstitute for Developmental Research, Aichi Human Service Center, Kasugai, Japan; ${ }^{6}$ RIKEN Tsukuba Institute, Tsukuba, Japan; ${ }^{7}$ Program of Developmental Neurobiology, National Institute of Child Health and Human Development, National Institute of Health, Bethesda, MD, USA; ${ }^{8}$ Department of Synthetic Chemistry and Biological Chemistry, Graduate School of Engineering, Kyoto University, Kyoto, Japan; ${ }^{9}$ Department of Pathology, Stanford University School of Medicine, Stanford, CA, USA
\end{abstract}

\begin{abstract}
Although the brains of patients with schizophrenia and bipolar disorder exhibit decreased brain $\mathrm{pH}$ relative to those of healthy controls upon postmortem examination, it remains controversial whether this finding reflects a primary feature of the diseases or is a result of confounding factors such as medication and agonal state. To date, systematic investigation of brain pH has not been undertaken using animal models that can be studied without confounds inherent in human studies. In the present study, we first reevaluated the $\mathrm{pH}$ of the postmortem brains of patients with schizophrenia and bipolar disorder by conducting a meta-analysis of existing data sets from 10 studies. We then measured $\mathrm{pH}$, lactate levels, and related metabolite levels in brain homogenates from five neurodevelopmental mouse models of psychiatric disorders, including schizophrenia, bipolar disorder, and autism spectrum disorder. All mice were drug naive with the same agonal state, postmortem interval, and age within each strain. Our meta-analysis revealed that brain pH was significantly lower in patients with schizophrenia and bipolar disorder than in control participants, even when a few potential confounding factors (postmortem interval, age, and history of antipsychotic use) were considered. In animal experiments, we observed significantly lower pH and higher lactate levels in the brains of model mice relative to controls, as well as a significant negative correlation between pH and lactate levels. Our findings suggest that lower $\mathrm{pH}$ associated with increased lactate levels is not a mere artifact, but rather implicated in the underlying pathophysiology of schizophrenia and bipolar disorder.

Neuropsychopharmacology (2018) 43, 459-468; doi:I0.1038/npp.2017.167; published online 6 September 2017
\end{abstract}

\section{INTRODUCTION}

Schizophrenia, bipolar disorder, and autism spectrum disorder (ASD) are highly heritable psychiatric conditions, with clinical features transcending diagnostic categories (Hyman, 2010; Insel et al, 2010). Accumulating evidence indicates that some genetic influences (Carroll and Owen, 2009; Cross-Disorder Group of the Psychiatric Genomics Consortium, 2013a, b; Lotan et al, 2014), gene expression abnormalities (Ellis et al, 2016; Shao and Vawter, 2008), and neuronal dysfunctions (Goodkind et al, 2015; Yahata et al, 2016) associated with these conditions overlap, suggesting a common underlying biological basis. However, the shared neurobiological alterations among the three conditions remain largely unknown.

*Correspondence: Dr T Miyakawa, Division of Systems Medical Science, Institute for Comprehensive Medical Science, Fujita Health University, Kutsukake-cho, Toyoake, Aichi 470- I 192, Japan, Tel: +8I 562939376 , Fax: +8I 56292 5382, E-mail: miyakawa@fujita-hu.ac.jp

Received 4 May 2017; revised 14 July 2017; accepted I August 2017; accepted article preview online 4 August 2017
A number of postmortem studies have indicated that $\mathrm{pH}$ is decreased in the brains of patients with schizophrenia and bipolar disorder (Guillozet-Bongaarts et al, 2014; Halim et al, 2008; Iwamoto et al, 2005; Lipska et al, 2006; Mistry et al, 2013; Prabakaran et al, 2004; Ryan et al, 2006; Shao and Vawter, 2008; Sun et al, 2006; Torrey et al, 2005). Decreased brain $\mathrm{pH}$ has also been observed in patients with ASD (Young et al, 2011). In general, $\mathrm{pH}$ balance is considered critical for maintaining optimal health, and low $\mathrm{pH}$ has been associated with a number of somatic disorders (Kraut and Madias, 2014; Narins and Emmett, 1980; Posner and Plum, 1967). Therefore, it is reasonable to assume that lower $\mathrm{pH}$ may exert a negative impact on brain function and play a key role in the pathogenesis of various psychiatric disorders. However, lower brain $\mathrm{pH}$ has largely been considered as an artifact rather than a pathophysiology of such disorders. Animal studies have indicated that chronic treatment with antipsychotics may affect brain $\mathrm{pH}$ by increasing lactate levels (Halim et al, 2008), and most patients with these disorders receive chronic antipsychotic treatment throughout their lives. In addition, the agonal state experienced 
before death decreases brain $\mathrm{pH}$ (Li et al, 2004; Tomita et al, 2004; Vawter et al, 2006), and this state may differ between patients with psychiatric disorders and controls. In human postmortem studies, it is technically difficult to exclude such confounding factors and to determine whether decreased $\mathrm{pH}$ and increased lactate levels are indeed artifacts.

In the present study, we first confirmed that patients with schizophrenia and bipolar disorder exhibit lower postmortem brain $\mathrm{pH}$ by conducting a meta-analysis of publicly available data sets. We then measured brain $\mathrm{pH}$ in multiple mouse models of psychiatric disorders, which are devoid of such confounding factors, in order to test the hypothesis that decreased brain $\mathrm{pH}$ is a pathophysiological manifestation/ endophenotype of these disorders rather than a mere artifact. We also measured lactate levels, increases in which have frequently been linked to decreased $\mathrm{pH}$ in the brains of patients with psychiatric disorders (Halim et al, 2008; Prabakaran et al, 2004; Stork and Renshaw, 2005). To our knowledge, the present study is the first to systematically evaluate $\mathrm{pH}$ and lactate levels in mouse models of psychiatric disorders that eliminate the confounds inherent in human studies.

We focused on mouse models of psychiatric disorders reported to exhibit neurodevelopmental abnormalities in the brain, a part of which stay at a pseudo-immature state (Hagihara et al, 2013). Specifically, we measured $\mathrm{pH}$, lactate, and related metabolite levels in the postmortem brains of the following mouse models: schnurri-2 (Shn2) knockout (KO) mice (Takao et al, 2013), forebrain-specific calcineurin (Cn) KO mice (Cottrell et al, 2013; Miyakawa et al, 2003; Suh et al, 2013; Zeng et al, 2001), and neurogranin (Nrgn) KO mice (Huang et al, 2006; Huang and Huang, 2012; Pak et al, 2000) as a model of schizophrenia; mice with heterozygous knockout of the calcium/calmodulin-dependent protein kinase II a (Camk2a HKO mice) (Hagihara et al, 2016; Yamasaki et al, 2008) as a model of bipolar disorder; and mice with heterozygous knockout of the long isoform of chromodomain helicase DNA-binding protein 8 (Chd8 HKO mice) (Katayama et al, 2016) as a model of ASD. These mouse strains are characterized by mutations in genes implicated in the respective disorders and exhibit molecular and behavioral abnormalities relevant to each condition, indicating good construct and face validities, respectively (as described in detail in the Supplementary Materials and Methods).

\section{MATERIALS AND METHODS}

\section{Human Data}

We obtained $\mathrm{pH}$ data of healthy individuals and patients with schizophrenia and bipolar disorder from the Stanley Medical Research Institute (SMRI) database (https://www. stanleygenomics.org). Duplicate data among studies in the database were eliminated. In addition, we comprehensively searched the National Center for Biotechnology Information Gene Expression Omnibus (NCBI GEO) database for studies reporting individual $\mathrm{pH}$ data using the following terms: schizophrenia, bipolar disorder, autism. We also used $\mathrm{pH}$ data for healthy individuals and patients with schizophrenia obtained from the New South Wales Brain Tissue Resource Centre schizophrenia cohort (NSWBTRC-SC) (Fillman et al,
2013), and from a study by Dean et al (2016). Altogether, 10 publicly available data sets were utilized in the present study (Supplementary Table S1): five schizophrenia data sets (GSE17612, GSE21935, GSE21138, NSWBTRC-SC (Fillman et al, 2013), and Dean et al, 2016)), one bipolar disorder data set (GSE5392), and four combined schizophrenia and bipolar disorder data sets (SMRI Collection A, SMRI Collection C, GSE35977, GSE53987). In addition, we used data regarding postmortem interval and age from these data sets, as well as data regarding medication from SMRI Collection A, SMRI Collection C, Dean et al, 2016, and GSE5392.

\section{Animals}

We measured $\mathrm{pH}$, lactate, and related metabolite levels in Shn2 KO mice (Takao et al, 2013) $(n=5,6$ (controls, mutants)), Cn KO mice (Cottrell et al, 2013; Miyakawa et al, 2003; Suh et al, 2013; Zeng et al, 2001) $(n=6,5)$, Nrgn KO mice (Huang et al, 2006; Huang and Huang, 2012; Pak et al, 2000) $(n=6,5)$, Camk2a HKO mice (Hagihara et al, 2016; Yamasaki et al, 2008) $(n=5,5)$, Chd8 HKO mice (Katayama et al, 2016) ( $n=5,5)$, disrupted-in-schizophrenia 1 (Disc1)L100P mutant mice $(n=6,6)$, Disc1-Q31L mutant mice $(n=6,6)$ (Shoji et al, 2012), voltage-gated calcium channel $\beta$-anchoring and -regulatory protein (Barp) KO mice (Nakao et al, 2015) $(n=10,10)$, and their corresponding control littermates. Shn2 KO and wild-type control mice were obtained by breeding heterozygotes with a C57BL/6J background and those with a BALB/cA background (Takao et al, 2013). All other strains were characterized by a C57BL/6J background. Both male and female mice were used in the present study, as no difference in $\mathrm{pH}$ has been observed between sexes (Catts et al, 2005). All mice were between 19 and 45 weeks of age, and no significant difference in age was observed between controls and mutants within each strain (Shn2 KO, $39.3 \pm 3.0$ weeks, controls (Con), 34.9 \pm 1.2 weeks, $P=0.19 ; C n \mathrm{KO}, 20.6 \pm 0.47$ weeks, Con, $20.6 \pm 0.36$ weeks, $P=0.92 ;$ Nrgn KO, $32.9 \pm 1.2$ weeks, Con, $31.0 \pm 0.14$ weeks, $P=0.12$; Camk2a HKO, $33.0 \pm 0.052$ weeks, Con, $36.2 \pm 1.9$ weeks, $P=0.13$; Chd8 HKO, 41.0 weeks, Con, 41.0 weeks; Disc1-L100P Mut, $29.0 \pm 0.41$ weeks, Con, $29.0 \pm 0.40$ weeks, $P=0.97$; Disc1-Q31L Mut, $36.1 \pm 0.60$ weeks, Con, $36.3 \pm 0.66$ weeks, $P=0.80$; Barp KO, 20.3 weeks, Con, 20.3 weeks). All animal experiments were approved by the institutional animal care and use committee of Fujita Health University, based on the Law for the Humane Treatment and Management of Animals and the Standards Relating to the Care and Management of Laboratory Animals and Relief of Pain. Every effort was made to minimize the number of animals used. Shn2 KO, Cn KO, Nrgn KO, Camk2a HKO, and Chd8 HKO mice-but not Disc1-L100P mutant and Disc1-Q31L mutant, or Barp KO mice-exhibit good construct and face validities for schizophrenia, bipolar disorder, and ASD. Further details are included in the Supplementary Materials and Methods (Supplementary Table S2).

\section{Measurement of $\mathrm{pH}$}

Mice were killed via cervical dislocation and decapitation, following which whole brains were removed. The brains were immediately frozen in liquid nitrogen and stored 
at $-80{ }^{\circ} \mathrm{C}$ until use. We measured brain $\mathrm{pH}$ as previously described (Catts et al, 2005; Halim et al, 2008). Briefly, mouse brains were homogenized using a tissue homogenizer equipped with a conical pestle in ice-cold distilled $\mathrm{H}_{2} \mathrm{O}(5 \mathrm{ml}$ per $500 \mathrm{mg}$ of tissue). The $\mathrm{pH}$ was measured using a $\mathrm{pH}$ meter (LAQUA F-72, Horiba Scientific, Kyoto, Japan) after a three-point calibration at $\mathrm{pH} 4.0, \mathrm{pH} 7.0$, and $\mathrm{pH}$ 9.0. The $\mathrm{pH}$ experiments were performed in triplicate for each sample, following which homogenates were immediately frozen and stored at $-80^{\circ} \mathrm{C}$ until required for further analyses.

\section{Lactate and Glucose Measurements}

The concentration of lactate in the brain homogenates was determined using a multi-assay analyzer (GM7 MicroStat; Analox Instruments, London, UK) in accordance with the manufacturer's instructions. In our prior tests using several samples, we loaded 5, 10, and $20 \mu \mathrm{l}$ of supernatant to the instrument, observing linear, volume-dependent increases in the measured values $\left(r^{2}>0.99\right)$. Based on these results, we used $20 \mu \mathrm{l}$ of supernatant for lactate measurements. Similarly, glucose concentrations in $20 \mu \mathrm{l}$ supernatant samples were determined using a multiassay analyzer following calibration with $10 \mathrm{mmol} / \mathrm{ml}$ glucose standard solution. To normalize the effects of differences in genetic background and age among strains, $Z$-scores for $\mathrm{pH}$ and lactate levels were calculated within each strain and used for the correlation analysis.

\section{Pyruvate Measurement}

Pyruvate concentrations in $20 \mu \mathrm{l}$ supernatant samples were determined using a pyruvate assay kit (BioVision, Mountain View, CA). The fluorescence intensities were measured using a microplate reader equipped with a spectrofluorometer (ARVO X, PerkinElmer).

\section{Adenosine Diphosphate/Adenosine Triphosphate (ADP/ ATP) Ratio}

An ADP/ATP Ratio Assay Kit (BioVision) was used to measure the ADP and ATP concentrations, in accordance with the manufacturer's instructions.

\section{Data Analysis}

Human data. We used all data obtained and conducted two-way analyses of variance (ANOVA) and covariance (ANCOVA) for $\mathrm{pH}$, factoring in diagnosis and data set using SAS Studio software version 3.5 (SAS Institute, Cary, NC). Tukey's honest significant difference (HSD) post hoc test after ANOVA or ANCOVA was also employed to assess the significance of differences between the mean values of diagnostic groups.

Because of differences in methods used to calculate equivalent doses among the four data sets (fluphenazine $v s$ chlorpromazine equivalent), we calculated $Z$-scores for lifetime antipsychotic use. $Z$-score transformation-a traditional method of data normalization for direct comparison between different samples and conditions-was applied for each antipsychotic equivalent value and $\mathrm{pH}$ value using individual participant data within each of four data sets, according to the following formula:

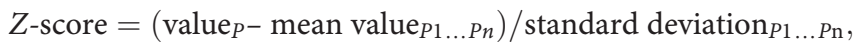
where $P$ is any $\mathrm{pH}$ and $P 1 \ldots \mathrm{P}_{n}$ represent the aggregate measure of all antipsychotic equivalent or $\mathrm{pH}$ values.

Mouse data. Student's $t$-test was employed to assess the significance of differences between the mean values of controls and mutants in combination with the BonferroniHolm correction for repeated measurements. Z-scores for $\mathrm{pH}$ and lactate levels were calculated within strains as described above.

\section{Transcriptome Analysis and Bioinformatics Analysis}

We used the following mouse brain transcriptome data: frontal cortex and hippocampal dentate gyrus (DG) of Shn2 KO mice (microarray) (Takao et al, 2013), hippocampal DG of Camk2a HKO mice (microarray) (Hagihara et al, 2009), and whole brains of Chd8 HKO mice (RNA-sequencing) (Katayama et al, 2016). Gene expression patterns in the frontal cortex of Camk2a HKO mice $(n=6,6)$ and hippocampal DG of $C n \mathrm{KO}$ mice $(n=6,6)$ were analyzed via microarray (Mouse Genome 430 2.0 Array; Affymetrix, Santa Clara, CA), as previously described (Takao et al, 2013). Gene expression patterns in the frontal cortex and hippocampal DG of Nrgn KO mice $(n=5,5)$ were analyzed via RNA-sequencing using the HiSeq platform in accordance with the manufacturer's instructions (Illumina, San Diego, CA). A total of eight transcriptome data sets were used in the present study. Genes with an absolute fold change $>1.2$ and a $t$-test $P$-value $<0.05$ (mutants $v s$ controls; without correction for multiple testing) were imported into the bioinformatics tool BaseSpace (Illumina), with which the gene expression data obtained from different platforms were matched (Hagihara et al, 2014). Genes meeting the above criteria are included in Supplementary Table S3. Genes with altered expression in at least four of the eight data sets (yielding 80 features; Supplementary Table S3) were selected based on the criteria of the BaseSpace tool and assessed for enrichment in biological themes using the DAVID functional annotation clustering tool, ADGO, and GOToolBox, in which the default feature listings and algorithm settings were used.

\section{RESULTS}

\section{Lower $\mathrm{pH}$ in the Postmortem Brains of Patients with Schizophrenia and Bipolar Disorder}

We first performed a meta-analysis of postmortem studies regarding brain $\mathrm{pH}$ in patients with schizophrenia and bipolar disorder that consisted of nine schizophrenia data sets and five bipolar disorder data sets (Figure 1 and Supplementary Table S1). A two-way ANOVA revealed significant effects of diagnosis $\left(\mathrm{F}_{2,694}=22.01, P=5.32 \times 10^{-10}\right)$ and data set $\left(\mathrm{F}_{9,694}=17.93, P<2.00 \times 10^{-16}\right)$, although no interaction was observed between the two factors $\left(F_{12,694}=1.70, P=0.063\right.$; Figure 1). The post hoc comparisons with Tukey's HSD test indicated that patients with schizophrenia (adjusted $P<1.0 \times 10^{-7}$ ) and bipolar disorder (adjusted $P=9.6 \times 10^{-6}$ ) 


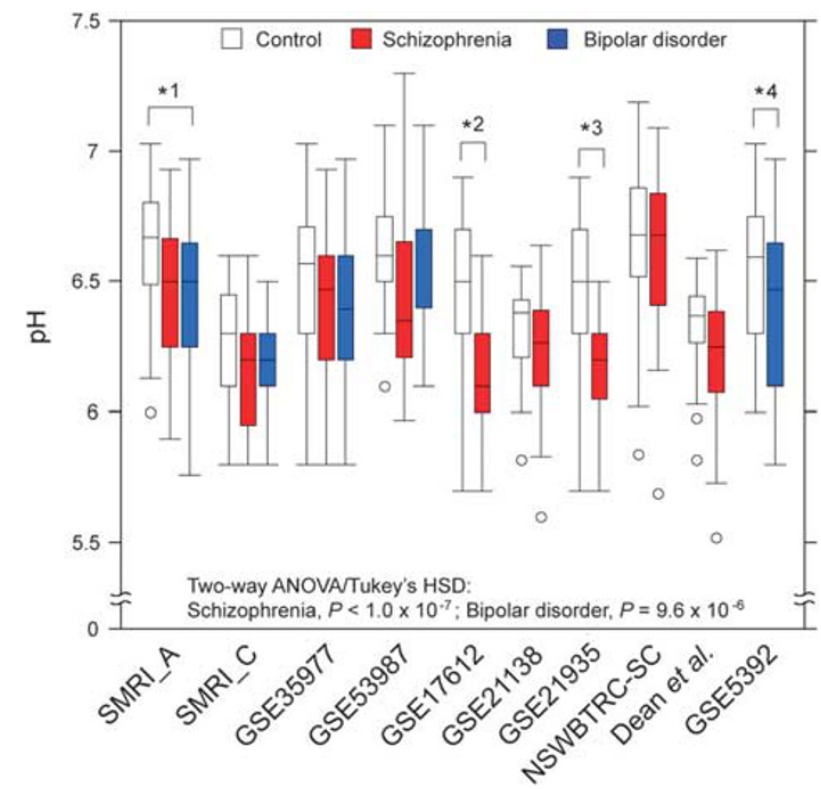

Figure I Lower $\mathrm{pH}$ in the postmortem brains of patients with schizophrenia and bipolar disorder. Box plot of brain $\mathrm{pH}$ in control participants (white box), patients with schizophrenia (red box), and patients with bipolar disorder (blue box). The boxes represent the interquartile range between first and third quartiles, whereas the whiskers represent the maximum and minimum values, and the circles represent population outliers. ${ }^{* 1} P=0.020,{ }^{* 2} P<0.000 I,{ }^{* 3} p=0.000 I,{ }^{* 4} p=0.027$; ANOVAV Tukey's post hoc test within each data set.

exhibited lower brain $\mathrm{pH}$ than healthy control. However, no significant difference in brain $\mathrm{pH}$ was observed between patients with schizophrenia and bipolar disorder (adjusted $P=0.88)$. Similar results were obtained with $Z$-score-transformed data: $\mathrm{pH}$ was significantly lower in patients with schizophrenia (adjusted $P<1.0 \times 10^{-7}$ ) and bipolar disorder (adjusted $P=1.59 \times 10^{-5}$ ) than in healthy controls, and no significant difference was observed between patients with schizophrenia and bipolar disorder (adjusted $P=0.85$ ) (Supplementary Figure S1).

We observed no significant interactions between diagnosis and postmortem interval/age in either schizophrenia (postmortem interval: $\mathrm{F}_{1,485}=2.56, P=0.11$; age: $\mathrm{F}_{1,485}=0.93$, $P=0.34$ ) or bipolar disorder data sets (postmortem interval: $\mathrm{F}_{1,295}=0.63, P=0.43$; age: $\mathrm{F}_{1,295}=1.07, P=0.30$ ). Hence, we performed correlation analyses between $\mathrm{pH}$ and such variables using the combined data of patients and controls to test their effects on $\mathrm{pH}$. In schizophrenia data sets, significant correlations were observed between $\mathrm{pH}$ values and age $(r=-0.17, P=0.00013)$, but not between $\mathrm{pH}$ and postmortem interval $(r=0.064, P=0.14)$. In bipolar disorder data sets, no significant correlations were observed between $\mathrm{pH}$ and postmortem interval $(r=0.072, P=0.20)$, or between $\mathrm{pH}$ and age $(r=0.032, P=0.57)$ (Supplementary Figure S2). Therefore, we performed an ANCOVA (factors: diagnosis and data set) on schizophrenia samples with age as a covariate that revealed a significant main effect of diagnosis $\left(\mathrm{F}_{1,485}=6.16, P=0.013\right)$. However, no significant interaction effects were observed for these factors $\left(\mathrm{F}_{8,485}=0.35\right.$, $P=0.95$ ). In bipolar disorder data sets, as no significant correlations were observed between $\mathrm{pH}$ and potential confounding factors, we performed an ANOVA to examine the effect of diagnosis on $\mathrm{pH}$. Our analysis revealed a significant main effect of diagnosis $\left(F_{1,305}=9.11\right.$, $P=0.0028)$, although no significant interaction effects were observed $\left(\mathrm{F}_{4,305}=0.68, P=0.60\right)$. The post hoc analyses revealed that $\mathrm{pH}$ was lower in the brains of patients with schizophrenia $(P<0.0001)$ and bipolar disorder $(P=0.0028)$ than in those of healthy controls. Furthermore, $Z$-scorebased meta-analysis of six data sets revealed no significant correlation between $\mathrm{pH}$ and lifetime use of antipsychotics $(r=-0.13, P=0.094$; Supplementary Figure S3), suggesting that antipsychotic treatment is not a major contributing factor affecting $\mathrm{pH}$ in the postmortem brains of patients with schizophrenia and bipolar disorder. Collectively, the results of our meta-analysis support the notion that lower brain $\mathrm{pH}$ is a pathological feature of schizophrenia and bipolar disorder rather than an artifact.

\section{Lower $\mathrm{pH}$ and Increased Lactate Levels in the Postmortem Brain of Mouse Models of Schizophrenia, Bipolar Disorder, and ASD}

The potential confounding factors identified in previous studies (Halim et al, 2008; Tomita et al, 2004) are beyond the investigator's control in postmortem studies of the human brain. We therefore measured $\mathrm{pH}$ and lactate levels in the brains of mouse models of schizophrenia (Shn2 KO, Cn KO, Nrgn KO mice), bipolar disorder (Camk2a HKO mice), and ASD (Chd8 HKO mice). All mice used were drug naive and killed via cervical dislocation, following which the removed brains were snap-frozen within a few minutes, allowing us to control for differences in agonal state and postmortem interval differences. Brain $\mathrm{pH}$ was significantly lower in all five mutant strains than in the corresponding controls (Shn2 KO, $7.17 \pm 0.0060$, Con, 7.20 $\pm 0.0056, P=0.017$; Cn KO, $7.08 \pm 0.0057$, Con, 7.13 $\pm 0.0080, P=0.0055 ; \quad N r g n$ KO, $7.10 \pm 0.017$, Con, 7.16 $\pm 0.0080, P=0.017$; Camk2a HKO, $7.14 \pm 0.0093$, Con, $7.21 \pm 0.0090, P=0.0055$; Chd $8 \mathrm{HKO}$, $7.08 \pm 0.0066$, Con, 7.12 $\pm 0.0031, P=0.0040$; Figure 2a).

Significantly higher levels of lactate were observed in the postmortem brains of all mutant mouse strains than in the corresponding controls (Shn2 KO, $2.98 \pm 0.080 \mathrm{mM}$, Con, $2.55 \pm 0.076 \mathrm{mM}, P=0.015 ; \mathrm{Cn} \mathrm{KO}, 3.24 \pm 0.051 \mathrm{mM}$, Con, $2.90 \pm 0.073 \mathrm{mM}, P=0.015 ; \mathrm{Nrgn} \mathrm{KO}, 2.98 \pm 0.11 \mathrm{mM}$, Con, $2.58 \pm 0.054 \mathrm{mM}, P=0.015$; Camk2a HKO, $2.86 \pm 0.024 \mathrm{mM}$, Con, 2.58 $\pm 0.037 \mathrm{mM}, P=0.0012$; Chd $8 \mathrm{HKO}, 3.04 \pm 0.081 \mathrm{mM}$, Con, $2.58 \pm 0.086 \mathrm{mM}, P=0.015$; Figure $2 \mathrm{~b}$ ). $Z$-score-based analysis revealed a significant negative correlation between $\mathrm{pH}$ and lactate levels (Pearson's $r=-0.65, P=1.19 \times 10^{-7}$; Figure 2c). In addition, lactate levels exhibited a significant negative correlation with age in the control group, but not in the mutant group (Supplementary Figure S4). However, there were no significant differences in age between controls and mutants of each strain (see the Materials and Methods). No significant correlations between age and $\mathrm{pH}$ were observed in either the mutant or control groups (Supplementary Figure S4).

Disc1-L100P mutant, Disc1-Q31L mutant, and Barp KO mice do not exhibit behavioral phenotypes associated with psychiatric disorders (Nakao et al, 2015; Shoji et al, 2012) (Supplementary Table S2). Unlike other model mice, mice of these three lines exhibited no changes in brain $\mathrm{pH}$ or lactate levels relative to those observed in the corresponding 

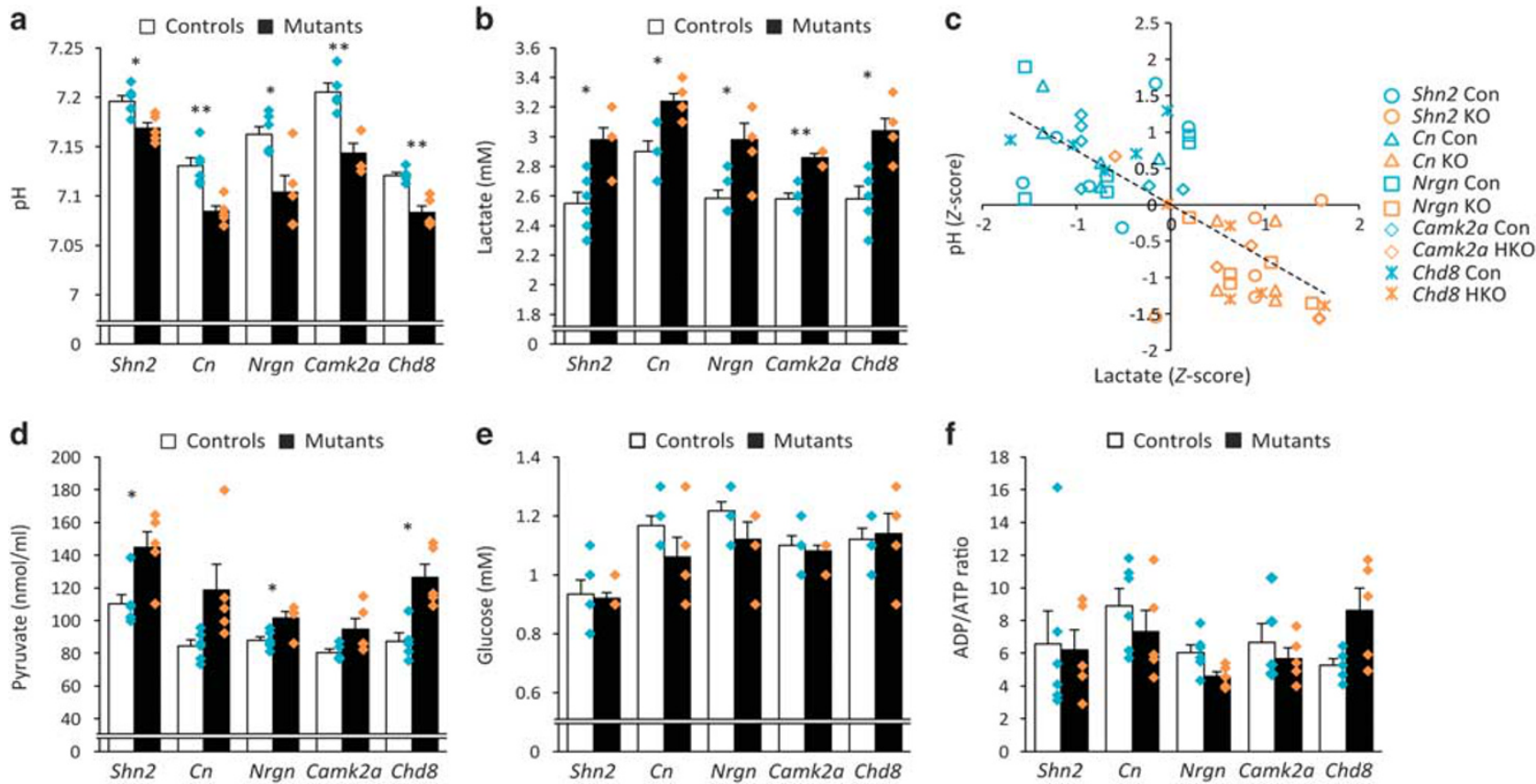

Figure 2 Lower $\mathrm{pH}$ and increased lactate levels in the brains of mouse models of psychiatric disorders. Bar graphs of pH (a), lactate levels (b), pyruvate levels (d), glucose levels (e), and ADP/ATP ratio ( $f$ in the brains of Shn2 KO, Cn KO, Nrgn KO, Camk2a HKO, and Chd8 HKO mice and their corresponding controls (mean \pm SEM). Each plot represents individual mouse values. (c) Scatter plot showing correlations between pH and lactate levels in the mouse brain. Asterisks indicate statistically significant differences between controls and mutants after Bonferroni-Holm correction $(* P<0.05$, $* * P<0.0$ I). ADP, adenosine diphosphate; ATP, adenosine triphosphate.

controls (Supplementary Figure S5). These results suggest that genetic alterations in general do not necessarily cause changes in $\mathrm{pH}$ and lactate levels in the mouse brain.

Lactate is formed from pyruvate during glycolysis. We therefore measured pyruvate levels in model mouse brains, observing that levels were significantly increased in Shn2 KO $(P=0.042)$, Nrgn KO $(P=0.042)$, and Chd8 HKO mice $(P=0.042)$. Cn KO $(P=0.092)$ and Camk2a HKO mice $(P=0.092)$ exhibited a trend toward increased pyruvate levels, although this trend did not survive Bonferroni-Holm correction (Figure 2d). Glucose levels remained unchanged in mutant mice relative to controls (Figure 2e), suggesting that glucose supply/demand ratio in the brain may be comparable in these mouse models. In addition, no significant differences in $\mathrm{ADP} / \mathrm{ATP}$ ratio were observed in these model mice following Bonferroni-Holm correction (Figure 2f), suggesting no alteration of energy consumption ratio in the brains of model mice.

We then analyzed transcriptome data (Supplementary Table S3) in order to investigate the potential underlying molecular mechanisms of increased lactate levels in mutant mouse brains. Transcriptome data from five mouse strains revealed an enrichment in Wnt- and epidermal growth factor (EGF)-related pathways when analyzed using DAVID software (Supplementary Table S4). Enrichment in Wnt-related pathways was replicated in analyses performed using other bioinformatics tools (ADGO and GOToolBox) using different statistical methods (Supplementary Table S4).

As lactate is produced via glycolytic pathways in astrocytes in the brain (Demetrius and Simon, 2012), we analyzed the transcriptome data of model mice with particular focus on glycolysis-related genes (Gene Ontology Consortium database) as well as those related to pyruvate metabolism. The results of the targeted gene expression analyses suggest that elevated glycolysis and pyruvate metabolism shifting toward lactate synthesis occur in the brains of model mice, especially in Shn2 KO and Camk2a HKO mice (Supplementary Table S5 and Supplementary Figure S6).

\section{DISCUSSION}

In the present study, our meta-analysis confirmed that the brains of patients with schizophrenia and bipolar disorder exhibit lower $\mathrm{pH}$ than healthy controls upon postmortem examination. Lower $\mathrm{pH}$ was also observed in five different mouse models of psychiatric disorders, all of which were drug naive and controlled for other potential confounding factors, such as agonal state and postmortem interval. We also observed increased lactate levels in the brains of model mice, as well as a highly significant negative correlation between $\mathrm{pH}$ and lactate levels, consistent with the findings of previous human postmortem studies (Halim et al, 2008). These results suggest that lower $\mathrm{pH}$ and increased lactate levels are implicated in the underlying pathophysiology of the diseases rather than mere artifacts.

Our meta-analyses indicated that brain $\mathrm{pH}$ is decreased in patients with schizophrenia and bipolar disorder when postmortem interval, age, and antipsychotic use are regarded as potential covariates of brain $\mathrm{pH}$. There are, however, still limitations regarding the covariates in the present study. One major limitation involves the lack of data regarding agonal state, as previous studies have reported that individuals who experience prolonged agonal states exhibit lower brain $\mathrm{pH}$ (Mexal et al, 2006; Tomita et al, 2004). These findings suggest that agonal state may be a potential confounding factor for postmortem brain $\mathrm{pH}$. As such, studies using 
animal models are necessary to validate the findings from human postmortem studies. However, as we cannot rule out the possibility that agonal states were altered in our model mice, further studies should examine $\mathrm{pH}$ and lactate levels in the brains of animals with short, moderate, and prolonged agonal states.

In addition to these limitations, other secondary factors cannot be ruled out in animal studies. For example, a recent study using Tbx1 mutant mice (ASD model mice) revealed that a genetic risk factor can influence maternal care via the phenotype of the risk carrier (Takahashi et al, 2016). Such secondary environmental factors during childhood may thus affect brain $\mathrm{pH}$ in mice. Increased locomotor activity may alter oxygen levels in the brains, particularly in Shn2 KO, Cn $\mathrm{KO}$, Nrgn KO, and Camk2a HKO mice (Supplementary Table S2). Considering that blood oxygen levels are positively correlated with brain lactate levels (Bednařík et al, 2015), oxygen levels may also represent a potential confounding factor in the measurement of brain lactate levels. Moreover, the balance between blood and brain cells may differ between the brain homogenates of control and mutant mice. As lactate concentrations in the blood are two- to three-fold higher than those in the mouse brain (extracellular compartment) (Béland-Millar et al, 2017), such differences may represent an additional confounding factor. Although differences in genetic background are also of concern when making comparisons between different mouse strains (Wolfer et al, 2002), we utilized over nine backcrossings in addition to littermates within each strain. Thus, it is unlikely that differences in $\mathrm{pH}$ and lactate levels between control and mutant mice within each strain reflect differences in genetic background.

We observed no significant differences in $\mathrm{pH}$ and lactate levels in the brain of two lines of Disc1 mutant mice as compared with corresponding controls. DISC1 has been implicated in the genetic etiology of schizophrenia (Brandon and Sawa, 2011; Ishizuka et al, 2006). However, a recent large-scale analysis of copy number variants (CNVs) suggested that DISC1 may not be a risk factor for the disorder (Marshall et al, 2016); the analysis showed that there are 5 deletions and 1 duplication in 21094 schizophrenia cases and 4 deletions and 2 duplications in 20227 controls (data deposited at UCSC Genome Browser on Human Mar. 2006, NCBI36/hg18). These results, combined with our previous observations that Disc1 mutant mice used in the present study did not exhibit behavioral phenotypes associated with schizophrenia (Shoji et al, 2012), suggest that these mutant mice may not represent a valid animal model of the disorder.

Increased lactate levels have been observed within certain brain regions in patients with schizophrenia (Halim et al, 2008; Prabakaran et al, 2004), bipolar disorder (Dager et al, 2004; Lan et al, 2008), and ASD (Goh et al, 2014) in both postmortem and in vivo spectroscopic imaging studies. More recent studies have confirmed increased lactate levels in the brains of patients with schizophrenia by postmortem analysis (Dean et $a l, 2016$ ) and in vivo analysis using 7Tesla magnetic resonance spectroscopy (MRS) (Rowland et al, 2016). Additional studies have revealed that pyruvate levels are increased whereas glucose levels remain unchanged in the postmortem brains of patients with schizophrenia (Dean et al, 2016). Our findings in mouse models are substantially consistent with the evidence obtained from previous human studies.

In our animal experiments, control baselines of $\mathrm{pH}$ and lactate levels varied among strains (Figures $2 \mathrm{a}$ and $\mathrm{b}$ ). Such variations may have been due to differences in age, as we observed a significant negative correlation between lactate levels and age in control mice; lactate levels increased as mouse age decreased (Supplementary Figure S4). These findings align with the neurodevelopmental hypothesis of schizophrenia. Accumulating evidence suggests that maturation abnormalities in certain types of brain cells, in which the molecular and physiological properties are similar to those of normal immature cells, represent an endophenotype commonly observed in several neuropsychiatric disorders, including schizophrenia, bipolar disorder, and epilepsy (Hagihara et al, 2013; Shin et al, 2013; Walton et al, 2012). For example, our previous study demonstrated that gene expression patterns in the prefrontal cortex of patients with schizophrenia are strikingly similar to those of typically developing infants (Hagihara et al, 2014). Considering the negative correlation between age and lactate levels in control mice of the present study, higher lactate levels in mutant mice may reflect one aspect of maturational abnormalities of the brain. However, additional studies involving detailed time-course analyses of developmental changes in $\mathrm{pH}$ and lactate levels are required to verify this hypothesis.

Previous studies have also revealed that brain acidosis influences a number of brain functions, such as anxiety, mood, and cognition (Wemmie, 2011). Acidosis may affect the structure and function of several types of brain cells, including the electrophysiological functioning of GABAergic neurons (Huang et al, 2015) and morphological properties of oligodendrocytes (Goldman et al, 1989). Alterations in these types of cells have been well documented in the brains of patients with schizophrenia, bipolar disorder, and ASD (Bartzokis, 2005; Nakazawa et al, 2012) and may underlie some of the cognitive deficits associated with these disorders. Deficits in GABAergic neurons and oligodendrocytes have also been identified in mouse models of the disorders, including Shn2 KO mice (Takao et al, 2013). Brain acidosis may therefore be associated with deficits in such cell types in schizophrenia, bipolar disorder, and ASD. However, as each genetic alteration may dysregulate the neurochemical balances of downstream molecules that are not functionally relevant to psychiatric disorders, further studies are required to determine whether low $\mathrm{pH}$ and increased lactate levels are functionally significant in psychiatric disorders.

A previous study indicated that chronic treatment with antipsychotics increases lactate levels in the rat cerebral cortex (Halim et al, 2008), suggesting that such increases may be medication related. The authors of the report, however, found no significant correlation between lactate levels and history of antipsychotic use in the post-mortem brains of patients with schizophrenia. In addition, increased lactate levels have been observed in the anterior cingulate of medication-free patients with bipolar disorder in in vivo spectroscopic imaging studies (Dager et al, 2004). Furthermore, studies utilizing animal models of psychiatric disorders have identified increased lactate levels in mutant mouse brains in vivo (das Neves Duarte et al, 2012). In addition, we observed an association between increased lactate levels and decreased $\mathrm{pH}$ in the brains of model mice 
in the present study, consistent with findings from previous studies on patients with schizophrenia (Halim et al, 2008; Prabakaran et al, 2004). Decreased brain $\mathrm{pH}$ has also been observed in medication-free patients with bipolar disorder (Kato et al, 1998). Although it is possible that antipsychotic treatment increases lactate levels and lowers $\mathrm{pH}$ in the brain, the aforementioned findings suggest that such changes may occur as primary features of schizophrenia and bipolar disorder.

It should be noted that haploinsufficiency of Chd8, a gene associated with ASD, results in lower brain $\mathrm{pH}$ and increased lactate levels in mice, as well as molecular and behavioral phenotypes relevant to ASD (Katayama et al, 2016). Previous studies have reported that patients with ASD exhibit decreased $\mathrm{pH}$ (Young et al, 2011) and increased lactate levels (Goh et al, 2014) relative to healthy controls, suggesting that our findings in model mice are consistent with those observed in patients with ASD. In addition, $\mathrm{CHD} 8$ has also been implicated in schizophrenia (Kimura et al, 2016; McCarthy et al, 2014). Therefore, lower $\mathrm{pH}$ and increased lactate levels in Chd8 HKO mice may reflect an aspect of brain pathophysiology in ASD/schizophrenia.

Interestingly, we observed that Wnt- and EGF-related pathways, which are highly implicated in somatic and brain cancers (Nicholas et al, 2006), are enriched in the genes whose expressions were altered among the five mutant mouse strains. It is well known that cancer cells display high rates of glycolysis, resulting in high lactate and pyruvate levels, even in normoxia (Lu et al, 2002). This phenomenon has been referred to as the Warburg effect. Genes whose expression is known to positively regulate the Warburg effect, such as Hk2 (Mathupala et al, 2009), Hif1a (Lu et al, 2002), and Pfkfb3 (Minchenko et al, 2002), were increased in the brains of some of mouse models examined in the present study, whereas expression of Prkaa1 - a negative regulator of the Warburg effect (Faubert et al, 2013)-was decreased (Supplementary Table S3). These findings suggest that elevated glycolysis underlies increases in lactate and pyruvate levels in the brains of schizophrenia, bipolar disorder, and ASD model mice. The results of the targeted gene expression analyses conducted in the present study also support this hypothesis. Glycolysis is also stimulated by the uptake of glutamate in astrocytes following neuronal excitation (Pellerin and Magistretti, 1994). Dysregulation of the excitation-inhibition balance has been proposed as a candidate cause of schizophrenia, bipolar disorder, and ASD (Brealy et al, 2015; Marín, 2012). A shift in the balance toward excitation would result in increased energy expenditure and may lead to increased glycolysis. Indeed, Shn2 KO mice exhibit higher glutamate levels in the hippocampus (Takao et al, 2013). In vivo metabolite measurements have suggested that increased glycolysis also occurs in the brains of patients with schizophrenia (Rowland et al, 2016) and bipolar disorder (Dager et al, 2004; Stork and Renshaw, 2005), whereas gene ontology analysis of microarray data has suggested that decreased glycolysis occurs in the brains of patients with schizophrenia (Prabakaran et al, 2004). Although further studies are required to determine whether alterations in the rate of glycolysis are associated with increased lactate levels and decreased $\mathrm{pH}$, we hypothesize that decreased $\mathrm{pH}$ in whole-brain samples was due to increased lactate production driven by hyperactivity within specific neural circuits (Hagihara et al, 2013; Heckers and Konradi, 2015; Lisman et al, 2008). Therefore, it would be of interest to investigate $\mathrm{pH}$ and lactate levels, as well as glycolysis rate, in the brains of mouse models of other mental disorders in which such hyperactivity has been implicated, such as epilepsy (Seifert and Steinhäuser, 2013), depression (Grace, 2016), and Alzheimer's disease (Busche et al, 2012).

Previous studies have indicated that lactate levels in the mouse brain rapidly increase after at least $1 \mathrm{~min}$ of decapitation, relative to those observed following in vivo fixation via focused microwave irradiation, regarded as a consequence of enhanced glycolysis under oxygen-deprived conditions (Sugiura et al, 2014). Although the current findings may differ from those obtained under physiological conditions, they may also reflect functional changes (eg, astrocyte activation) (Huang and Huang, 2012; Takao et al, 2013) that represent the main source of lactate production in the brain.

Brain $\mathrm{pH}$ is associated with notable changes in gene expression (Catts et al, 2005; Iwamoto et al, 2005; Mexal et al, 2006; Tomita et al, 2004) and has hence been considered as a confound for investigating changes in gene expression related to the pathophysiology of psychiatric disorders. Therefore, substantial effort has been made to match tissue $\mathrm{pH}$ between patients and controls. Given that lower brain $\mathrm{pH}$ is a pathophysiological component of certain conditions, $\mathrm{pH}$-dependent changes in gene expression are of concern when attempting to elucidate the molecular basis of the conditions. Some studies have indicated that gene expression patterns are partially similar across diseases such as schizophrenia, bipolar disorder, and ASD (Ellis et al, 2016; Shao and Vawter, 2008). Decreased $\mathrm{pH}$ may underlie these similarities in the pattern of gene expression. Thus, $\mathrm{pH}$ may be an important factor in the elucidation of molecular alternations in the brains of patients with these psychiatric conditions.

\section{FUNDING AND DISCLOSURE}

TM received research grants from Astellas Pharma Inc. CSW is on an advisory board for Lundbeck, Australia Pty Ltd and in collaboration with Astellas Pharma Inc. CSW is funded by the NSW Ministry of Health, Office of Health and Medical Research. CSW is a recipient of a National Health and Medical Research Council (Australia) Principal Research Fellowship (PRF) (\#1117079). CSW and VSC received tissues from the New South Wales Brain Tissue Resource Centre at the University of Sydney which is supported by the University of Sydney and The Schizophrenia Research Institute (Fillman et al, 2013). Research reported in this publication was supported by the National Institute of Alcohol Abuse and Alcoholism of the National Institutes of Health under Award Number R28AA012725. The content is solely the responsibility of the authors and does not represent the official views of the National Institutes of Health.

\section{ACKNOWLEDGMENTS}

We thank Gerald R Crabtree of Stanford University for providing $C n$ mutant mice, Wakako Hasegawa, Yumiko 
Mobayashi, Misako Murai, Tamaki Murakami, Miwa Takeuchi, Aki Miyakawa, Chikako Ozeki, Yuya Ouchi, Satoko Hattori, Toshiki Kameyama, Hidehito Inagaki, and Hiroki Kurahashi of Fujita Health University for their technical support in this study, as well as Yuki Sugiura of Keio University for helpful discussion. This work was supported by the Grants-in-Aid for Scientific Research (JP25242078) from Japan Society for the Promotion of Science; the Grant-in-Aid for Scientific Research on Innovative Areas "Unraveling the microendophenotypes of psychiatric disorders at the molecular, cellular and circuit levels" (JP25116526, JP15H01297) and "Dynamic regulation of brain function by Scrap \& Build system" (JP16H06462) from the Ministry of Education, Culture, Sports, Science and Technology; and the Strategic Research Program for Brain Sciences from Japan Agency for Medical Research and Development, AMED.

\section{REFERENCES}

Bartzokis G (2005). Brain myelination in prevalent neuropsychiatric developmental disorders. Adolesc Psychiatry 29: 55-96.

Bednařík P, Tkáč I, Giove F, DiNuzzo M, Deelchand DK, Emir UE et al (2015). Neurochemical and BOLD responses during neuronal activation measured in the human visual cortex at 7 Tesla. J Cereb Blood Flow Metab 35: 601-610.

Béland-Millar A, Larcher J, Courtemanche J, Yuan T, Messier C (2017). Effects of systemic metabolic fuels on glucose and lactate levels in the brain extracellular compartment of the mouse. Front Neurosci 11: 7.

Brandon NJ, Sawa A (2011). Linking neurodevelopmental and synaptic theories of mental illness via DISC1. Nat Rev Neurosci 12: 707-722.

Brealy JA, Shaw A, Richardson H, Singh KD, Muthukumaraswamy SD, Keedwell PA (2015). Increased visual gamma power in schizoaffective bipolar disorder. Psychol Med 45: 783-794.

Busche MA, Chen X, Henning HA, Reichwald J, Staufenbiel M, Sakmann B et al (2012). Critical role of soluble amyloid- $\beta$ for early hippocampal hyperactivity in a mouse model of Alzheimer's disease. Proc Natl Acad Sci USA 109: 8740-8745.

Carroll LS, Owen MJ (2009). Genetic overlap between autism, schizophrenia and bipolar disorder. Genome Med 1: 102.

Catts VS, Catts SV, Fernandez HR, Taylor JM, Coulson EJ, LutzeMann LH (2005). A microarray study of post-mortem mRNA degradation in mouse brain tissue. Mol Brain Res 138: 164-177.

Cottrell JR, Levenson JM, Kim SH, Gibson HE, Richardson KA, Sivula $M$ et al (2013). Working memory impairment in calcineurin knock-out mice is associated with alterations in synaptic vesicle cycling and disruption of high-frequency synaptic and network activity in prefrontal cortex. J Neurosci 33: 10938-10949.

Cross-Disorder Group of the Psychiatric Genomics Consortium (2013a). Genetic relationship between five psychiatric disorders estimated from genome-wide SNPs. Nat Genet 45: 984-994.

Cross-Disorder Group of the Psychiatric Genomics Consortium (2013b). Identification of risk loci with shared effects on five major psychiatric disorders: a genome-wide analysis. Lancet $\mathbf{3 8 1}$ : 1371-1379.

Dager SR, Friedman SD, Parow A, Demopulos C, Stoll AL, Lyoo IK et al (2004). Brain metabolic alterations in medication-free patients with bipolar disorder. Arch Gen Psychiatry 61: 450-458.

das Neves Duarte JM, Kulak A, Gholam-Razaee MM, Cuenod M, Gruetter R, Do KQ (2012). N-acetylcysteine normalizes neurochemical changes in the glutathione-deficient schizophrenia mouse model during development. Biol Psychiatry 71: 1006-1014.
Dean B, Thomas N, Scarr E, Udawela M (2016). Evidence for impaired glucose metabolism in the striatum, obtained postmortem, from some subjects with schizophrenia. Transl Psychiatry 6: e949.

Demetrius LA, Simon DK (2012). An inverse-Warburg effect and the origin of Alzheimer's disease. Biogerontology 13: 583-594.

Ellis SE, Panitch R, West AB, Arking DE (2016). Transcriptome analysis of cortical tissue reveals shared sets of downregulated genes in autism and schizophrenia. Transl Psychiatry 6: e817.

Faubert B, Boily G, Izreig S, Griss T, Samborska B, Dong Z et al (2013). AMPK is a negative regulator of the Warburg Effect and suppresses tumor growth in vivo. Cell Metab 17: 113-124.

Fillman SG, Cloonan N, Catts VS, Miller LC, Wong J, McCrossin T et al (2013). Increased inflammatory markers identified in the dorsolateral prefrontal cortex of individuals with schizophrenia. Mol Psychiatry 18: 206-214.

Goh S, Dong Z, Zhang Y, DiMauro S, Peterson BS (2014). Mitochondrial dysfunction as a neurobiological subtype of autism spectrum disorder: evidence from brain imaging. JAMA Psychiatry 71: 665-671.

Goldman SA, Pulsinelli WA, Clarke WY, Kraig RP, Plum F (1989). The effects of extracellular acidosis on neurons and glia in vitro. J Cereb Blood Flow Metab 9: 471-477.

Goodkind M, Eickhoff SB, Oathes DJ, Jiang Y, Chang A, Jones-Hagata LB et al (2015). Identification of a common neurobiological substrate for mental illness. JAMA Psychiatry 72: 305-315.

Grace AA (2016). Dysregulation of the dopamine system in the pathophysiology of schizophrenia and depression. Nat Rev Neurosci 17: 524-532.

Guillozet-Bongaarts AL, Hyde TM, Dalley RA, Hawrylycz MJ, Henry A, Hof PR et al (2014). Altered gene expression in the dorsolateral prefrontal cortex of individuals with schizophrenia. Mol Psychiatry 19: 478-485.

Hagihara H, Horikawa T, Nakamura HK, Umemori J, Shoji H, Kamitani $\mathrm{Y}$ et al (2016). Circadian gene circuitry predicts hyperactive behavior in a mood disorder mouse model. Cell Rep 14: 2784-2796.

Hagihara H, Ohira K, Takao K, Miyakawa T (2014). Transcriptomic evidence for immaturity of the prefrontal cortex in patients with schizophrenia. Mol Brain 7: 41.

Hagihara H, Takao K, Walton NM, Matsumoto M, Miyakawa T (2013). Immature dentate gyrus: an endophenotype of neuropsychiatric disorders. Neural Plast 2013: e318596.

Hagihara H, Toyama K, Yamasaki N, Miyakawa T (2009). Dissection of hippocampal dentate gyrus from adult mouse. J Vis Exp 33 pii: 1543.

Halim ND, Lipska BK, Hyde TM, Deep-Soboslay A, Saylor EM, Herman $\mathrm{M}$ et al (2008). Increased lactate levels and reduced $\mathrm{pH}$ in postmortem brains of schizophrenics: medication confounds. J Neurosci Methods 169: 208-213.

Heckers S, Konradi C (2015). GABAergic mechanisms of hippocampal hyperactivity in schizophrenia. Schizophr Res 167: 4-11.

Huang FL, Huang K-P (2012). Methylphenidate improves the behavioral and cognitive deficits of neurogranin knockout mice. Genes Brain Behav 11: 794-805.

Huang FL, Huang K-P, Wu J, Boucheron C (2006). Environmental enrichment enhances neurogranin expression and hippocampal learning and memory but fails to rescue the impairments of neurogranin null mutant mice. J Neurosci 26: 6230-6237.

Huang L, Zhao S, Lu W, Guan S, Zhu Y, Wang J-H (2015). Acidosis-induced dysfunction of cortical GABAergic neurons through astrocyte-related excitotoxicity. PLoS ONE 10: e0140324.

Hyman SE (2010). The diagnosis of mental disorders: the problem of reification. Annu Rev Clin Psychol 6: 155-179.

Insel T, Cuthbert B, Garvey M, Heinssen R, Pine DS, Quinn K et al (2010). Research domain criteria (RDoC): toward a new 
classification framework for research on mental disorders. Am J Psychiatry 167: 748-751.

Ishizuka K, Paek M, Kamiya A, Sawa A (2006). A review of Disrupted-in-Schizophrenia-1 (disc1): neurodevelopment, cognition, and mental conditions. Biol Psychiatry 59: 1189-1197.

Iwamoto K, Bundo M, Kato T (2005). Altered expression of mitochondria-related genes in postmortem brains of patients with bipolar disorder or schizophrenia, as revealed by large-scale DNA microarray analysis. Hum Mol Genet 14: 241-253.

Katayama Y, Nishiyama M, Shoji H, Ohkawa Y, Kawamura A, Sato $\mathrm{T}$ et al (2016). CHD8 haploinsufficiency results in autistic-like phenotypes in mice. Nature 537: 675-679.

Kato T, Murashita J, Kamiya A, Shioiri T, Kato N, Inubushi T (1998). Decreased brain intracellular pH measured by 31P-MRS in bipolar disorder: a confirmation in drug-free patients and correlation with white matter hyperintensity. Eur Arch Psychiatry Clin Neurosci 248: 301-306.

Kimura H, Wang C, Ishizuka K, Xing J, Takasaki Y, Kushima I et al (2016). Identification of a rare variant in CHD8 that contributes to schizophrenia and autism spectrum disorder susceptibility. Schizophr Res 178: 104-106.

Kraut JA, Madias NE (2014). Lactic acidosis. N Engl J Med 371: 2309-2319.

Lan MJ, McLoughlin GA, Griffin JL, Tsang TM, Huang JTJ, Yuan P et al (2008). Metabonomic analysis identifies molecular changes associated with the pathophysiology and drug treatment of bipolar disorder. Mol Psychiatry 14: 269-279.

Li JZ, Vawter MP, Walsh DM, Tomita H, Evans SJ, Choudary PV et al (2004). Systematic changes in gene expression in postmortem human brains associated with tissue $\mathrm{pH}$ and terminal medical conditions. Hum Mol Genet 13: 609-616.

Lipska BK, Deep-Soboslay A, Weickert CS, Hyde TM, Martin CE, Herman MM et al (2006). Critical factors in gene expression in postmortem human brain: focus on studies in schizophrenia. Biol Psychiatry 60: 650-658.

Lisman JE, Coyle JT, Green RW, Javitt DC, Benes FM, Heckers S et al (2008). Circuit-based framework for understanding neurotransmitter and risk gene interactions in schizophrenia. Trends Neurosci 31: 234-242.

Lotan A, Fenckova M, Bralten J, Alttoa A, Dixson L, Williams RW et al (2014). Neuroinformatic analyses of common and distinct genetic components associated with major neuropsychiatric disorders. Front Neurosci 8: 331.

Lu H, Forbes RA, Verma A (2002). Hypoxia-inducible factor 1 activation by aerobic glycolysis implicates the Warburg effect in carcinogenesis. J Biol Chem 277: 23111-23115.

Marshall CR, Howrigan DP, Merico D, Thiruvahindrapuram B, Wu W, Greer DS et al (2016). Contribution of copy number variants to schizophrenia from a genome-wide study of 41,321 subjects. Nat Genet 49: 27-35.

Marín O (2012). Interneuron dysfunction in psychiatric disorders. Nat Rev Neurosci 13: 107-120.

Mathupala SP, Ko YH, Pedersen PL (2009). Hexokinase-2 bound to mitochondria: cancer's stygian link to the "Warburg effect" and a pivotal target for effective therapy. Semin Cancer Biol 19: 17-24.

McCarthy SE, Gillis J, Kramer M, Lihm J, Yoon S, Berstein Y et al (2014). De novo mutations in schizophrenia implicate chromatin remodeling and support a genetic overlap with autism and intellectual disability. Mol Psychiatry 19: 652-658.

Mexal S, Berger R, Adams CE, Ross RG, Freedman R, Leonard S (2006). Brain $\mathrm{pH}$ has a significant impact on human postmortem hippocampal gene expression profiles. Brain Res 1106: 1-11.

Minchenko A, Leshchinsky I, Opentanova I, Sang N, Srinivas V, Armstead V et al (2002). Hypoxia-inducible factor-1-mediated expression of the 6-phosphofructo-2-kinase/fructose-2,6-bisphosphatase-3 (PFKFB3) gene. Its possible role in the Warburg effect. J Biol Chem 277: 6183-6187.
Mistry M, Gillis J, Pavlidis P (2013). Meta-analysis of gene coexpression networks in the post-mortem prefrontal cortex of patients with schizophrenia and unaffected controls. BMC Neurosci 14: 105.

Miyakawa T, Leiter LM, Gerber DJ, Gainetdinov RR, Sotnikova TD, Zeng $\mathrm{H}$ et al (2003). Conditional calcineurin knockout mice exhibit multiple abnormal behaviors related to schizophrenia. Proc Natl Acad Sci USA 100: 8987-8992.

Nakao A, Miki T, Shoji H, Nishi M, Takeshima H, Miyakawa T et al (2015). Comprehensive behavioral analysis of voltage-gated calcium channel beta-anchoring and -regulatory protein knockout mice. Front Behav Neurosci 9: 141.

Nakazawa K, Zsiros V, Jiang Z, Nakao K, Kolata S, Zhang S et al (2012). GABAergic interneuron origin of schizophrenia pathophysiology. Neuropharmacology 62: 1574-1583.

Narins RG, Emmett M (1980). Simple and mixed acid-base disorders: a practical approach. Medicine (Baltimore) 59: 161-187.

Nicholas MK, Lukas RV, Jafri NF, Faoro L, Salgia R (2006). Epidermal growth factor receptor-mediated signal transduction in the development and therapy of gliomas. Am Assoc Cancer Res 12: 7261-7270.

Pak JH, Huang FL, Li J, Balschun D, Reymann KG, Chiang C et al (2000). Involvement of neurogranin in the modulation of calcium/calmodulin-dependent protein kinase II, synaptic plasticity, and spatial learning: a study with knockout mice. Proc Natl Acad Sci USA 97: 11232-11237.

Pellerin L, Magistretti PJ (1994). Glutamate uptake into astrocytes stimulates aerobic glycolysis: a mechanism coupling neuronal activity to glucose utilization. Proc Natl Acad Sci USA 91: 10625-10629.

Posner JB, Plum F (1967). Spinal-fluid $\mathrm{pH}$ and neurologic symptoms in systemic acidosis. N Engl J Med 277: 605-613.

Prabakaran S, Swatton JE, Ryan MM, Huffaker SJ, Huang JT-J, Griffin JL et al (2004). Mitochondrial dysfunction in schizophrenia: evidence for compromised brain metabolism and oxidative stress. Mol Psychiatry 9: 684-697 643.

Rowland LM, Pradhan S, Korenic S, Wijtenburg SA, Hong LE, Edden RA et al (2016). Elevated brain lactate in schizophrenia: a 7 $\mathrm{T}$ magnetic resonance spectroscopy study. Transl Psychiatry 6: e967.

Ryan MM, Lockstone HE, Huffaker SJ, Wayland MT, Webster MJ, Bahn S (2006). Gene expression analysis of bipolar disorder reveals downregulation of the ubiquitin cycle and alterations in synaptic genes. Mol Psychiatry 11: 965-978.

Seifert G, Steinhäuser C (2013). Neuron-astrocyte signaling and epilepsy. Exp Neurol 244: 4-10.

Shao L, Vawter MP (2008). Shared gene expression alterations in schizophrenia and bipolar disorder. Biol Psychiatry 64: 89-97.

Shin R, Kobayashi K, Hagihara H, Kogan JH, Miyake S, Tajinda K et al (2013). The immature dentate gyrus represents a shared phenotype of mouse models of epilepsy and psychiatric disease. Bipolar Disord 15: 405-421.

Shoji H, Toyama K, Takamiya Y, Wakana S, Gondo Y, Miyakawa T (2012). Comprehensive behavioral analysis of ENU-induced Disc1-Q31L and -L100P mutant mice. BMC Res Notes 5: 108.

Stork C, Renshaw PF (2005). Mitochondrial dysfunction in bipolar disorder: evidence from magnetic resonance spectroscopy research. Mol Psychiatry 10: 900-919.

Sugiura Y, Honda K, Kajimura M, Suematsu M (2014). Visualization and quantification of cerebral metabolic fluxes of glucose in awake mice. Proteomics 14: 829-838.

Suh J, Foster DJ, Davoudi H, Wilson MA, Tonegawa S (2013). Impaired hippocampal ripple-associated replay in a mouse model of schizophrenia. Neuron 80: 484-493.

Sun X, Wang J-F, Tseng M, Young LT (2006). Downregulation in components of the mitochondrial electron transport chain in the postmortem frontal cortex of subjects with bipolar disorder. $J$ Psychiatry Neurosci 31: 189-196. 
Takahashi T, Okabe S, Broin PÓ, Nishi A, Ye K, Beckert MV et al (2016). Structure and function of neonatal social communication in a genetic mouse model of autism. Mol Psychiatry 21: 1208-1214.

Takao K, Kobayashi K, Hagihara H, Ohira K, Shoji H, Hattori S et al (2013). Deficiency of Schnurri-2, an MHC enhancer binding protein, induces mild chronic inflammation in the brain and confers molecular, neuronal, and behavioral phenotypes related to schizophrenia. Neuropsychopharmacology 38: 1409-1425.

Tomita H, Vawter MP, Walsh DM, Evans SJ, Choudary PV, Li J et al (2004). Effect of agonal and postmortem factors on gene expression profile: quality control in microarray analyses of postmortem human brain. Biol Psychiatry 55: 346-352.

Torrey EF, Barci BM, Webster MJ, Bartko JJ, Meador-Woodruff JH, Knable MB (2005). Neurochemical markers for schizophrenia, bipolar disorder, and major depression in postmortem brains. Biol Psychiatry 57: 252-260.

Vawter M, Tomita H, Meng F, Bolstad B, Li J, Evans S et al (2006). Mitochondrial-related gene expression changes are sensitive to agonal-pH state: implications for brain disorders. Mol Psychiatry 11: 615-679.

Walton NM, Zhou Y, Kogan JH, Shin R, Webster M, Gross AK et al (2012). Detection of an immature dentate gyrus feature in human schizophrenia/bipolar patients. Transl Psychiatry 2: e135.

Wemmie JA (2011). Neurobiology of panic and $\mathrm{pH}$ chemosensation in the brain. Dialogues Clin Neurosci 13: 475-483.

Wolfer DP, Crusio WE, Lipp H-P (2002). Knockout mice: simple solutions to the problems of genetic background and flanking genes. Trends Neurosci 25: 336-340.
Yahata N, Morimoto J, Hashimoto R, Lisi G, Shibata K, Kawakubo Y et al (2016). A small number of abnormal brain connections predicts adult autism spectrum disorder. Nat Commun 7: 11254.

Yamasaki N, Maekawa M, Kobayashi K, Kajii Y, Maeda J, Soma M et al (2008). Alpha-CaMKII deficiency causes immature dentate gyrus, a novel candidate endophenotype of psychiatric disorders. Mol Brain 1: 6.

Young A, Campbell E, Lynch S, Suckling J, Powis S (2011). Aberrant NF-kappaB expression in autism spectrum condition: a mechanism for neuroinflammation. Front Psychiatry 2: 27.

Zeng H, Chattarji S, Barbarosie M, Rondi-Reig L, Philpot BD, Miyakawa T et al (2001). Forebrain-specific calcineurin knockout selectively impairs bidirectional synaptic plasticity and working/ episodic-like memory. Cell 107: 617-629.

This work is licensed under a Creative Commons

4.0
International License. The images or other third party material in this article are included in the article's Creative Commons license, unless indicated otherwise in the credit line; if the material is not included under the Creative Commons license, users will need to obtain permission from the license holder to reproduce the material. To view a copy of this license, visit http://creativecommons.org/licenses/by-nc-nd/4.0/

(C) The Author(s) 2018

Supplementary Information accompanies the paper on the Neuropsychopharmacology website (http://www.nature.com/npp) 